\title{
Modelling agent societies: co-ordination frameworks and institutions
}

\author{
Virginia Dignum $^{1,2}$, Frank Dignum ${ }^{2}$ \\ ${ }^{1}$ Achmea \\ PO Box 866, 3700 AW Zeist, The Netherlands \\ virginia.dignum@achmea.nl \\ ${ }^{2}$ University Utrecht \\ Institute for Information and Computing Sciences \\ PO Box 80.089, 3508TB Utrecht, The Netherlands \\ dignumecs.uu.nl
}

\begin{abstract}
Organisations can be defined as a set of entities regulated by mechanisms of social order and created by more or less autonomous actors to achieve common goals. Multi-agent systems are a natural choice to design information support systems due to the proactive and autonomous behaviour of agents. However, in business environments it is necessary to consider the behaviour of the global system and the collective aspects of the domain. In this paper, we argue that multi-agent systems should be designed around organisational co-ordination frameworks that reflect the co-ordination structures of the particular organisation. As in human societies, we argue that norms and institutions are a way for agent societies to cope with the challenge of social order. Through institutions, conventions and interaction patterns for the co-ordination of agents can be specified, monitored and managed.
\end{abstract}

Keywords: Agent societies, co-ordination, institutions, virtual organisations 


\title{
Modelling agent societies: co-ordination frameworks and institutions
}

\author{
Virginia Dignum $^{1,2}$, Frank Dignum ${ }^{2}$
}

\section{Introduction}

Software agents, characterised as autonomous entities with reasoning and communicative capabilities, are utmost suitable to implement, simulate or represent real-life entities presenting the same autonomy. Because of the proactive and autonomous behaviour of agents it is natural to design information support systems using agent societies that mimic the behaviour and structure of human organisations [23]. Agent societies represent the interactions between agents and are as such the virtual counterpart of real-life societies and organisations. Agents model specific roles in the society and interact with others as a means to accomplish their goals. This perspective makes the design of the system less complex since it reduces the conceptual distance between the system and the real-world application it has to model. Therefore, agent societies are an effective platform for virtual organisations because they provide mechanisms to allow organisations to advertise their capabilities, negotiate their terms, exchange rich information, and synchronise processes and workflow at a high-level of abstraction [18].

An organisation can be seen as a set of entities regulated by mechanisms of social order and created by more or less autonomous actors to achieve common goals. In business environments the behaviour of the global system and the collective aspects of the domain need to be considered. That is, design must be able to consider the collective characteristics of the organisation such as stability over time, some level of predictability, and clear commitment to aims and strategies. However, typically, agents are assumed to pursue their own individual goals and global behaviour emerges from individual interactions. Existing architectures, behavioural strategies and models for group formation often assume this individualist perspective.

In this paper, we argue that multi-agent systems that are developed to model and support organisations have to have co-ordination frameworks that mimic the coordination structures of the particular organisation. Therefore methodologies for designing such multi-agent systems have to be able to describe different types of coordination models. As in human societies, we argue that norms and institutions are a way for agent societies to cope with the challenge of social order. In section 2 we introduce a design methodology for agent societies that is based on the structural characteristics of an organisation and described by different co-ordination frameworks. The role of institutions in the engineering of agent societies is described in section 3. In section 4, we present some practical applications being developed at Achmea. Finally, in section 5 we give some conclusions and directions for future work. 


\section{Organisational multi-agent systems}

There is a rising awareness that multi-agent systems and cyber-societies can best be understood and developed if they are inspired by human social phenomena [1, 5, 23]. Virtual organisations consist of independently developed components that may change, join or leave the virtual organisation over time. That is, virtual organisations are open societies and therefore agent societies modelling virtual organisations cannot be designed based solely on the internal architectures of its members. Such societies are expected to form a coherent, stable system that realises the objectives for which it was designed. Therefore any formalism for the design of open agent societies must fulfil the following requirements:

- Agent societies must describe roles, norms and goals of the society instead of just agent states.

- Mechanisms are needed to verify whether the design of an agent society satisfies its design requirements and objectives [15]

- Interactions between members and the expected outcome of roles must be formalised in order to verify the overall animation of the society

- The organisational and normative elements of a society must be explicitly specified since an open society cannot rely on its embedding in the intentions, desires and beliefs of each agent [7, 17]

- Communication and ability to conform to expected role behaviour are assumed for members.

Furthermore, in order to facilitate the development of organisation oriented multiagent systems it is necessary to relate to the organisational perception of the problem. That is, a common ground of understanding must be found between agent engineers and organisational practitioners. In our opinion co-ordination is an ideal candidate. In one hand, organisational science and economics have since long researched coordination and organisational structures. Relationships between and within organisations are developed for the exchange of goods, resources, information and so on. Depending on transaction costs and interdependent relations, different coordination models (market, hierarchy or network) are possible. On the other hand, coordination is one of the cornerstones of agent societies and is considered an important problem inherent to the design and implementation of MAS [2]. However, the implications of the co-ordination model for the agent society architecture and design method have usually not been considered. So far, research about co-ordination in MAS has been mainly limited to the study of technical aspects of co-ordination, such as control and planning. In many cases the social organisation is left implicit in the design of the agent society.

\subsection{Methodology for the development of agent societies}

Multi agent systems are often viewed as aggregations of agents that interact with each other [13]. In this individualistic perspective, co-ordination emerges from patterns of agent actions as result of interaction. It is therefore not possible to impose any requirements and objectives to co-ordination. In the organisational view of multiagent systems design, we are interested in the collectivist view: co-ordination is 
designed through pre-established roles, responsibilities and norms. The type of organisational structure determines important autonomous activities, which must be explicitly organised into autonomous entities and relationships in the conceptual model of the agent society [11].

Domain requirements for any system can be divided into functional (what is the system suppose to do) and interaction (how is the system expected to do it) requirements. Co-ordination structures define roles and interactions specific to an organisational structure (market, hierarchy or network) and are determined by interaction. We have developed a methodology (described in more detail in [11]) for the design of agent societies based on the type of co-ordination structure. In general terms, the aim of the methodology is to provide generic facilitation and interaction frameworks for agent societies that implement the functionality derived from the coordination model applicable to the problem domain. These frameworks are described in section 2.2.

The first step of the methodology is to support and orient the choice of a coordination model based on the interaction requirements of the domain. In the next stage, the ontological requirements of the society and the requirements for interaction with the environment are specified Finally, the functional requirements are used to extend the co-ordination framework with domain specific roles and interaction forms that characterise the problem. This process results in an exemplary society, which behaviour and animation can be verified and the compliance to domain requirements can be checked.

We can compare this process to the design a generic enterprise model including roles as accountants, secretaries and managers, as well as their job descriptions and relationships, and then extending it with the functions necessary to achieve the objectives of the given enterprise. These are, for example, designers and carpenters if the firm is going to manufacture chairs, and programmers and system analysts if the enterprise is a software house.

\subsection{Co-ordination models}

Following the basic classification of co-ordination models from organisational theory, co-ordination in agent societies can be divided into markets, networks and hierarchies. Different co-ordination models result in different frameworks for agent societies. The overall goals of a society are domain dependent but all societies depend on a facilitation layer that provides the social backbone of the organisation. The objectives of the facilitation layer are the organisation of the society itself and are dependent on the underlying co-ordination model and on the norms and conventions that hold in the domain. Social co-ordination describes the way interactions between roles are organised and the way the interface between the society and the 'outside world' is defined. That is, the co-ordination model determines the institutional roles, social norms and interaction forms in the society. Table 1 gives an overview of the characteristics of different agent societies.

In the remainder of this section we will introduce the facilitation roles associated with each co-ordination model. In section 3 , the norms and conventions needed to regulate interaction and specify the desired behaviour of its members will be described. 


\begin{tabular}{|l|c|c|c|}
\hline Type of society & Market & Network & Hierarchy \\
\hline $\begin{array}{l}\text { Members } \\
\text { 'values' }\end{array}$ & Open & Trust & Closed \\
\hline $\begin{array}{l}\text { Society } \\
\text { purpose }\end{array}$ & Exchange & Collaboration & Production \\
\hline Interaction & $\begin{array}{c}\text { Interaction is based on } \\
\text { standards; communication } \\
\text { concerns exchange only }\end{array}$ & $\begin{array}{c}\text { Both the interaction } \\
\text { procedures and } \\
\text { exchange can be } \\
\text { negotiated }\end{array}$ & $\begin{array}{c}\text { Specified on } \\
\text { design }\end{array}$ \\
\hline
\end{tabular}

Table 1. Characteristics of agent societies

The characteristics and requisites for each role will determine the required capabilities of the agents that have to fulfil the role, in terms of its communicative and reasoning capabilities. For example, agents acting in a network are expected to negotiate their interaction procedures and are motivated by mutual interest. This means such agents will be required to be able to reason about other agents and need to possess 'heavy' negotiation algorithms. On the other hand, members of a hierarchical society follow pre-determined communication lines and have limited need for negotiation, thus agents fulfilling hierarchical roles can be much simpler in terms of communication and negotiation capabilities. In the following, we will describe each co-ordination model in more detail, indicating necessary facilitation roles and its characteristics and providing examples of existing multi-agent systems that follow that model.

In order to be able to assign roles to agents, we need to make some assumptions on the capabilities of the agent. However, because we must be able to apply society models to different, possibly open, domains it is not possible to make too many assumptions on the specific architecture of agents. Therefore, we choose to use the generic model illustrated in figure 1 as a basis for our assumption on agents. Generic agent models have been introduced in [4]. The interpretation of the components of an agent is as follows:

- Control and Planning. Agents must be able to organise their own behaviour. We will not expand on this aspect, however, it is something that all agents need, whichever role they will perform.

- Interaction with world. Describes the ways an agent can interact with the world, depending on the demands of its role. Includes sensors and interfaces to databases and other applications.

- Interaction with agents. Describes the communicative capabilities of an agent.

- Goals. Describes the goals of the agent.

- Tasks. Describes the specific tasks of the agent. Existing task models can be used for further specification of this component

- World knowledge. Processes involved with maintaining the agents knowledge, including knowledge about the environment and knowledge of other agents abilities 


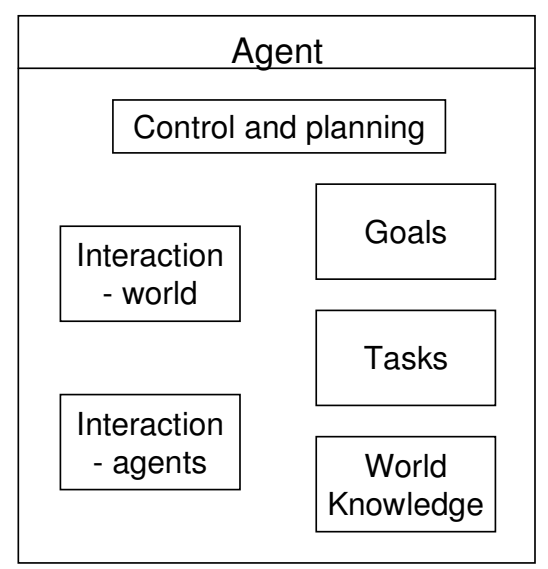

Fig. 1. Generic Agent Model

This model makes no demands on the way each component is designed, but assumes that agents will in some way be able to perform the indicated components. Specific agents are free to design their own internal components in different ways, and even do without some of the components. The description of roles will indicate what are the society expectations on the capabilities of agents that perform the role, in terms of these components.

\subsubsection{Roles in the Market co-ordination model}

The main goal of a market is to facilitate exchange between agents. In a market model, agents are self-interested (determine and follow their own goals), represent (or provide) services and/or competencies and compete to perform tasks leading to the satisfaction of their own individual objectives. Agents are usually assumed to be heterogeneous and the negotiation rules are fixed (for example Contact Net or Dutch auction). Interaction in markets occurs through communication and negotiation with the market rules.

Co-ordination through a market mechanism is particularly well suitable for situations in which resources can be described easily or are commoditised, there are several agents offering the same (type) of resources and several agents that need them. Besides obvious e-commerce applications, the market architecture is also a good choice to model product or service allocation problems. Being self-interested, agents will first try to solve their own local problem, and then agents can potentially negotiate with other agents to exchange services or goods in shortage or in excess. Agent societies based on the market model have been used to represent virtual enterprises [19]. Facilitation roles necessary for the organisation of a market model are:

- Identification: has the task of registering members of the society. Can also receive requests from matchmakers or bankers

- Matchmaker: keeps track of agents in the system, their needs and possibilities and mediates in the matching of demand and supply of goods or services. Depending 
on the domain, the task of a matchmaker can be a simple unification algorithm or a complex fuzzy matching algorithm. Matchmakers must be able to receive requests from agents and contact possible partners. Depending on the domain, this capabilities can be just a simple message request(buyer?, product, price) or announce(seller, product, price) or it can involve more general communication determining the requirements on both products and potential partner. Furthermore, matchmakers need to have knowledge of current sellers and requests in the society. I.e. they need to maintain a kind of yellow guide.

- Banking: define ways to value the goods to be exchanged and determine profit and fairness of exchanges. A banking service builds confidence for customers as well as offers guarantees to the members of the society. Bankers must be able to receive requests from agents wishing to register themselves (open an account) or wishing to get information on other agents, and need to keep knowledge on their clients

\subsubsection{Roles in the Hierarchy co-ordination model}

Hierarchies co-ordinate the flow of resources or information by controlling and directing it at a central point in the managerial hierarchy. Managerial decisions and not negotiation and communication as in markets determine interaction and design. Demand parties do not select a supplier from a group of potential suppliers: they simply work with a predetermined one. In hierarchical systems, each agent controls a statically defined sub-hierarchy (possibly empty), in many cases an administrative domain of some kind. Environments where the workflow is fixed and cases are repetitive, such as in automated manufacturing are well suited to the hierarchical model. In such systems, reliable control of resources and information flow requires central entities that manage local resources and data but also need quick access to global ones. For instance, a university could be managed as follows: an agent is in charge in each lab, whereas other agents each oversee a department and a single one rules the university. Hierarchical models of agents have been used to model information agents ([6]) and the management of communication networks ([14]).

In a hierarchical co-ordination model, agents at facilitation level are mainly dedicated to the overall control and optimisation of the system activities. Sometimes, these facilitation activities are concentrated in one agent, typically the 'root' agent of the hierarchy. Facilitation roles necessary to the organisation of a hierarchy are:

- Controllers: monitor and orient the overall performance of the system or of a part of the system. Autonomous agents have local perspective and their actions are determined by its local state. Therefore, in a hierarchical co-ordination model it is necessary to have an agent whose role is to control the overall performance of the system.

- Interface agents: are responsible for the communication between the system and the 'outside world'. In this architecture communication lines between agents are predefined. Furthermore, agents are usually not free to enter or leave the system. Therefore communication with the outside world must be regulated at the facilitation level. 


\subsubsection{Roles in the Network co-ordination model}

Networks are coalitions of self-interested agents that agree to collaborate to achieve a mutual goal. Agents in a network society are self-interested but are willing to trade some of their freedom to obtain secure relations and trust. Network coordination models are built around general patterns of interaction or contracts. Relationships are dependent on clear communication patterns and social norms. Coordination is achieved by mutual interest, possibly using trusted third parties, and according to well-defined rules and sanctions. These coalitions have been studied in the area of game theory and Distributed Artificial Intelligence (DAI) [21]. Dellarocas introduces the concept of Contractual Agent Societies (CAS) as a model for developing agent societies [7].

Network co-ordination models provide an explicit shared context, describing rules and social norms for interaction and collaboration. The society is responsible to make its rules and norms known to potential members. Agents in a network society enter a social contract with the society in which they commit themselves to act within and according to the norms and rules of the society.

At the facilitation level of a network, agents monitor, register and help others form contracts, introduce (teach) new agents to the rules of the market and keep track of the reputation of agents. Furthermore, they keep and enforce the 'norms' of the agent community and ensure interaction. Roles at facilitation level in networks are:

- Matchmaker: keeps track of agents in the system, their needs and possibilities and mediates in the matching of demand and supply of goods or services. In the network co-ordination domain, the matching of supply and demand is usually more complex than in markets, because long-term interests have to be taken into account. Therefore, matchmakers will need to use, for instance, fuzzy matching algorithms, or multi-attribute matching to be able to perform their tasks. As in markets, matchmakers must be able to receive requests from agents and contact possible partners and need to keep knowledge of current offers and requests in the society.

- Gatekeeper: is responsible for accepting and introducing new agents to the market. Agents entering the marketplace must be informed about the possibilities and capabilities of the market. Gatekeepers negotiate the terms of a social contract between the applicant and the members of the market.

- Notary: register collaboration contracts between agents.

- Monitoring agents: are trusted third parties that keep track of the execution of collaboration contracts between agents.

\section{The role of institutions}

Usually human organisations and societies use norms and conventions to cope with the challenge of social order. Norms and conventions specify the behaviour that society members are expected to conform to and are suitable for decentralised control. In most societies, norms are backed by a variety of social institutions that enforce law and order (e.g. courts, police), monitor for and respond to emergencies (e.g. ambulance system), prevent and recover from unanticipated disasters (e.g. coast guard, fire-fighters), etc. In this way civilised societies allow citizens to utilise 
relatively simple and efficient rules of behaviour, offloading the prevention and recovery of many problem types to social institutions that can handle them efficiently and effectively by virtue of their economies of scale and widely accepted legitimacy. Successful civil societies have thus achieved a division of labour between individuals and institutions that decreases the "barriers to survival" for each citizen, while helping increase the welfare of the society as a whole [20].

Several researchers have recognised that the design of agent societies can benefit from abstractions analogous to those employed by our robust and relatively successful societies and organisations. There is a growing body of work that touches upon the concepts of norms and institutions in the context of multi-agent systems (cf. [9, 10, 12]).

The benefit of an institution resides in its potential to lend legitimacy and security to its members by establishing norms. The electronic counterpart of the physical institution does a similar task for software agents: it can engender trust through certification of an agent and by the guarantees that it provides to back collaboration. However, the electronic institution can also function as the independent place in which al types of agent independent information about the interaction between the agents within the society is stored. E.g. it defines the message types that can be used by the agents in their interactions, the rules of encounter, etc. In general, institutions enable to:

- Specify the co-ordination structure that is used

- Describe exchange mechanisms of the agent society

- Determine interaction and communication forms within the agent society

- Facilitate the perception of individual agents of the aims and norms of an agent society

- Enforce the organisational aims of the agent society

In our approach we consider that an agent society consists of two layers: one is facilitation-oriented and the other goal-oriented. The institution acts as mediator and animator for the members, who bring various skills and services, and customers (or groups of customers) who bring their problems and requirements. The most important service the institution provides is to regulate the interaction between members. Because the way interaction between agents happens depends on the co-ordination model, institutions will need to be defined differently for each co-ordination model.

We have shown above that co-ordination models provide a setting for agent societies by setting out the goals of the society and the roles (what you can do) need to achieve those goals. Institutions will enforce this model by setting out the scenes (where you can do it) and protocols (what you can say) for interaction in the society. This defines how agents can interact with the institution or with other agents in the society. The whole point of institutions is for the additional services it can provide and the trust and guarantees that are established through the institution's credibility and norms.

Looking at the structure of organisations we can anticipate the types of interaction involved in interacting in a particular co-ordination model. Thus, an institution defines a performative structure and a dialogical framework, by which we mean, it prescribes the actions members can take and when and where to perform those actions, and determines the form of conversations between members. Therefore, the way norms and conventions are specified and enforced in a society depends on the co- 
ordination model. In hierarchies, norms and conventions can be embedded in the power relations. These relations determine which agent can demand an action from which other agent or which agent has priority over the resources. The controlling agent is supposed to uphold the norms of the society by managing the sub-ordinate agents according to them. In markets, norms and conventions are for a large part embedded in the market mechanism chosen. E.g. the auction mechanisms try to ensure that all agents get an opportunity to require a resource relative to their private value for that resource. Cheating by over- or underbidding does not lead to any benefit for the agent and thus is prevented by the mechanism itself. In network models explicit roles are defined to 'represent' the institution that enforce monitoring and trust, and trace the fulfilment of contracts. Some examples of these roles will be given in the next section.

\section{Applications}

The framework described in this paper can be applied to very distinct problem domains, because it concentrates on the organisational elements of the agent societies. At Achmea, a financial and insurance holding organisation operating mainly in the Netherlands, the ideas described in this paper are being applied to the development of a system for support of knowledge sharing (K-Exchange), and in the area of secondary healthcare co-ordination (CareMarket).

Achmea strives to the optimal support of clients both in their primary and secondary health care needs. Furthermore we are concerned with the development of communities and the involvement of individuals in their community (neighbourhood, company, etc.). The aim of the community care project CareMarket is to provide Achmea clients with extra (unskilled) care services, which are not covered by professional organisations, or for which there are long waiting lists. Such services are, for example: getting medicines, help with household chores during recovery, transport to doctor/hospital, preparation of meals, babysitting (while the mother is visiting the doctor). The project is inspired by the LETS concept and based on non-monetary trading concepts. Matching of supply and demand in this kind of situations is not trivial. The fulfilment of a demand usually requires the co-ordination of several suppliers, suppliers are voluntaries and usually of a very limited and constrained range of services. Furthermore, it is desirable to keep a continuity of relationships between suppliers and clients (people tend to develop friendship relations with their care tenders / care takers and do not really appreciate to see a new face every day).

This pilot is in a very initial phase of development but there is already a clear realisation that the institutional framework described in this paper will be directly applicable to the development of an agent-based simulation prototype. The evaluation of the system through the simulated institution populated with intelligent agents, representing suppliers and clients, will provide insights and support to the eventual deployment of a real community pilot.

The Knowledge Exchange Network system is in a more advanced phase of development and will be described in more detail in the next section. 


\subsection{Knowledge Exchange Network}

The objective of the Knowledge Exchange Network project is to support non-life insurance experts to exchange knowledge with each other, in a way that preserves the knowledge, rewards the knowledge owner and reaches the knowledge seeker in a justin-time, just-enough basis. Current users of the pilot project are project managers, product developers, actuaries in the Non-life group of Achmea but in the future it will be extended to other people (e.g. call centre employees) and groups. Members of the network have lots of knowledge, which is greatly valuable and useful to each other. So, one of the main tasks of the Knowledge Exchange Network is be to support and encourage their contacts. Experience shows that any technological support for knowledge exchange greatly improves if users feel they know and can trust each other. Therefore, the Knowledge Management activities at the Non-life group consist of two parts:

- Quarterly face-to-face workshops with the aim of getting people to know each other, share their experiences and extend their knowledge (through invited speakers, theme discussions, etc.)

- Development of a virtual network, aiming both at a knowledge repository and at the support of communication and collaboration

The virtual network, whose pilot is now under development using the intranet of Achmea, aims at preserving knowledge and facilitating sharing. For the share support module, an agent society is being developed using the framework based design method described in this paper. Sharing is not centrally controlled but greatly encouraged. Both knowledge seekers and knowledge owners act as though in a market: they decide where to 'buy', where to 'sell', what, to whom and under which conditions. The best-suited partner, according to each participant's own conditions, will get the job. Moreover, even within one organisation, factors such as privacy, secrecy and competitiveness between brands and departments may influence the channels and possibilities of sharing and must thus be considered.

The following requirements expressed by the project stakeholders are used to determine both the applicability of an agent-based solution and the desirable type of co-ordination:

- The organisation aims at supporting collaboration and extending synergy. Another aim of the organisation is to preserve existing knowledge and make it available organisation-wide.

- Knowledge owners are willing to share their knowledge within a controllable, trusted group; they wish therefore, to be able to control their own sharing decisions and conditions; added-value of the sharing effort and fair exchange is a must (that is, the feeling that one is rewarded for share must be achieved)

- Knowledge seekers are not aware of existing knowledge and its whereabouts and who are possible knowledge owners; as knowledge owners, they also wish to be able to decide on acquisition conditions and partners; furthermore an accreditation and certification mechanism is desired, that enables checking the level of trust and knowledge of partners

These requirements identify a distributed system where different actors, acting autonomously on behalf of a user and each pursuing its own goals, need to interact in order to achieve their goals. Thus, communication and negotiation are paramount. 
Furthermore, the number and behaviour of participants, and the type, quantity and quality of the goods cannot be fixed a priori and the system can be expected to expand and change during operation. These characteristics indicate that the agent society frameworks described in this paper are well suitable.

Considering the requirements of the stakeholders described above, the network model is the most appropriate for this situation. The aim is to design an exchange society restricted to selected participants with the global goal of supporting collaboration and synergy, and in this way meet the organisation requirements. Participants are aware of and collaborative with this requirement but also have their own objectives and constraints. Participants wish to be free to determine their own exchange rules and to be assured that there is control over who are the other participants in the environment.

Due to space limitations, we cannot describe the complete system in this paper. In the following we will describe some of the roles and interactions. Having decided for a network structure, the roles of matchmaker, notary, monitor, and gatekeeper follow naturally from the application of the framework. From the domain requirements the roles of knowledge owner and knowledge seeker can be deduced. The 'goods' to be exchanged are the contents of the knowledge repository, that is, (XML) documents representing knowledge about reports, people, applications, web sites, projects, questions, etc. ${ }^{1}$ Figure 2, shows a fragment of the architecture of the society, indicating roles and possible interaction procedures. These procedures are also determined by the model chosen (network). The interactions represented in Figure 2 are informally described below. It is important to note that:

- Interactions are usually multi-action processes and not one single action. That is, the result is usually only achieved after a conversation. Similar to what in [12] is referred to as a scene, an interaction between agents is defined through conversations following a well-defined protocol.

- Interactions are described for the binary case (one seeker, one owner). However, it is possible to form multi partner contracts.

- $\quad \mathrm{X}$ represents any agent and $\mathrm{M}$ and $\mathrm{N}$ represent any members of the society

\footnotetext{
1 This type of goods demands a complex matching mechanism, since matches are not at keyword level but require knowledge about relationships, processes etc. This imposes constraints to the task and communicative components of agents. This will not be discussed here.
} 


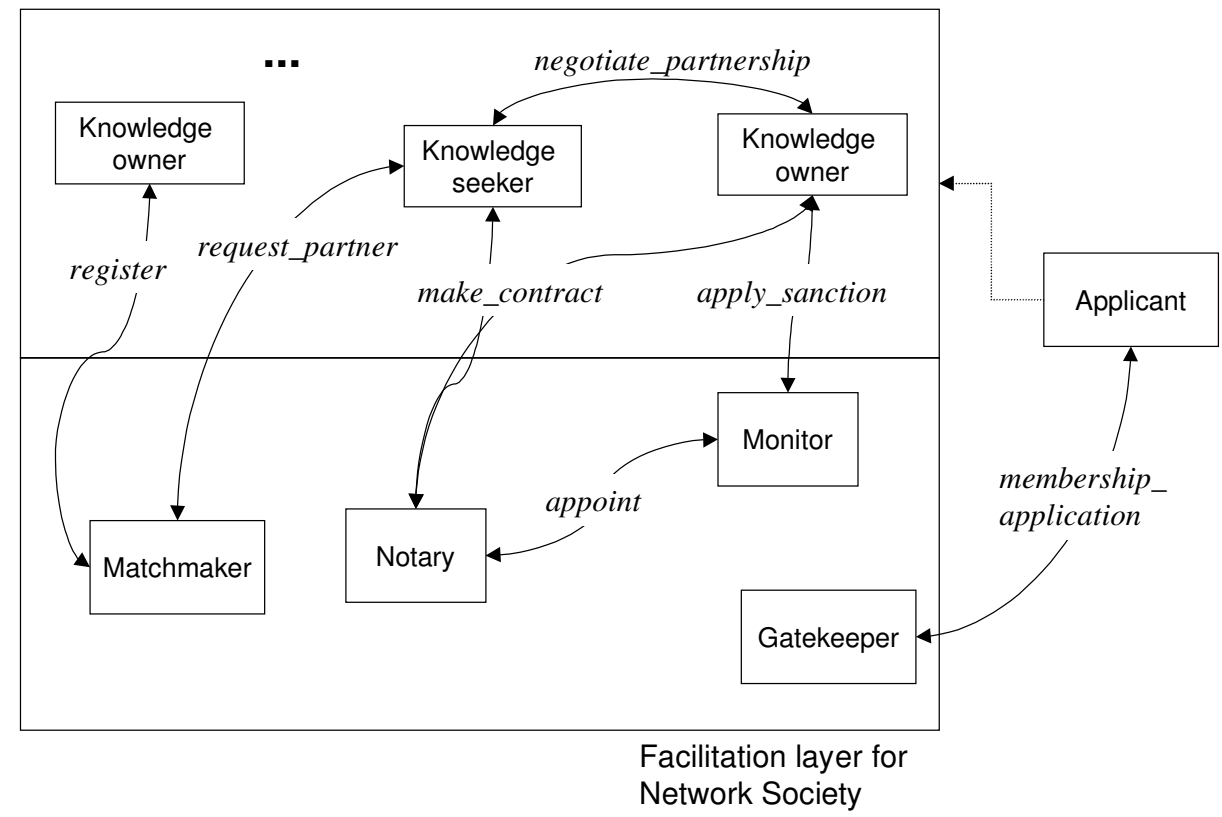

Fig. 2. Fragment of the Knowledge Exchange Network architecture

1. membership_application( $X$, gatekeeper): This is a negotiation between any agent and the gatekeeper of the society resulting in either an acceptance, that is $\mathrm{X}$ will become member of the society, or a rejection. The role the agent will play is also determined in this scene.

2. register( $M$, matchmaker): Knowledge owners or seekers can register their requests with the matchmaker, who will use this information in future matches

3. request_partner( $M$, matchmaker): Knowledge owners or seekers request possible partners for an exchange. Results in a possibly empty list of potential partners.

4. negotiate_partnership $(M, N)$ : Owners and seekers check the viability of an exchange and determine conditions

5. make_contract $(M, N$, notary): When an agreement is reached, partners register their commitments with the notary.

6. appoint(notary, monitor): the notary appoints a monitor for a contract. It delegates agreed tasks to the monitor. The monitor will keep track of contract status and will act when an undesired state is reached.

7. apply_sanction(monitor, $M$ ): when a breech of contract occurs the monitor will contact the faulty party and apply the sanctions agreed upon (either described in the contract or standard in the institution).

The institution underlying the society will impose mechanisms for collaboration and certification. For instance, a special kind of knowledge owner is responsible for the gathering and dissemination of information on a known, fixed list of competitors to knowledge seekers interested. The institution will in this case enforce the norm that 
such agents are required to provide all the information they are aware of. This determines the task for the monitors tracing this type of contracts of checking if information in all companies in the list is indeed provided.

\section{Conclusions and future work}

We have presented a framework for the design of agent societies based on the coordination structure of the domain that uses institutions to specify and enforce social norms and conventions. The framework takes the organisational perspective as starting point. We believe that one contribution of our research is that it describes the implications of the co-ordination model of the organisation for the architecture and design method of the agent society being developed. Although there are several agentbased software engineering methodologies (see, $[8,3,16,22])$ these are often either too specific or too formal and not easily used and accepted. Our approach is to provide a generic frame that directly relates to the organisational perception of a problem. If needed, existing methodologies can be used for the development, modelling and formalisation of each step. We believe that our approach will contribute to the acceptance of multi-agent technology by organisations.

Institutions play an important role to specify and manage the conventions of the agent society. One of the most important aspects is that they can make organisational goals and norms explicit and warrant their fulfilment.

We are currently applying the ideas described in this paper to develop a Knowledge Exchange Network at Achmea and we plan to use it to develop a system for agent based mediation for health care. Feedback from these applications will be used to improve the design methodology and the co-ordination frameworks used.

Important work that is left for the future is the formal description of both the coordination framework as well as the institutions. This will provide means for verifying properties of the institution. It will also enable agents that consider joining the society whether they are able and willing to conform to the specified conventions and interaction mechanisms.

\section{References}

1. Artikis, A., Kamara, L., Pitt, J.: Towards an Open Agent Society Model and Animation, Proceedings of the Agent-Based Simulation II workshop, Passau, (2001) 48-55

2. Bond, A., Gasser, L.: Readings in Distributed Artificial Intelligence. Morgan Kaufmann, (1988)

3. Brazier, F., Dunin-Keplicz, B., Jennings, N., Treur, J.: DESIRE: Modelling Multi-Agent Systems in a Compositional Formal Framework. In: Huhns, M., Singh M. (eds.): International Journal of Cooperative Information Systems, 6(1) (1997)

4. Brazier, F., Jonker, C., Treur, J.: Compositional Design and Reuse of a Generic Agent Model. Applied Artificial Intelligence Journal,14, (2000) 491-538.

5. Castelfranchi, C.: Engineering Social Order, Omicini, A., Tolksdorf, R., Zambonelli, F., (Eds.) Engineering Societies in the Agents World, First International Workshop, ESAW 2000, Berlin, Germany, LNAI 1972, Springer-Verlag (2000) 1 - 19 
6. Castillo, A., Kawaguchi, M., Paciorek, N., Wong, D.: Concordia ${ }^{\mathrm{TM}}$ as Enabling Technology for Cooperative Information Gathering. In: Proceedings of Japanese Society for Artificial Intelligence Conference, Tokyo, Japan (1998)

7. Dellarocas, C.: Contractual Agent Societies: Negotiated shared context and social control in open multi-agent systems. In: Proceedings of Workshop on Norms and Institutions in Multi-Agent Systems, Autonomous Agents-2000, Barcelona (2000)

8. DeLoach, S.: Multiagent Systems Engineering: A Methodology and Language for Designing Agent Systems. In: Proceedings of Workshop on Agent-Oriented Information Systems (AOIS'99) (1999)

9. Dignum, F.: Autonomous Agents with Norms. In AI and Law, (7), (1999) 69 - 79.

10. Dignum, F.: Agents, Markets, Institutions and Protocols. In Dignum, F., Sierra, C. (eds.): Agent Mediated Electronic Commerce, LNAI 1991, Springer-Verlag (2001) 98 - 114.

11. Dignum, V., Weigand, H., Xu L.: Agent Societies: Towards framework-based design. In: Wooldridge, M., Ciancarini P., Weiss, G. (Eds.): Proceedings of the 2nd Workshop on Agent-Oriented Software Engineering, Autonomous Agents, Montreal, (2001) 25-31.

12. Esteva, M., Padget, J., Sierra, C.: Formalizing a language for Institutions and Norms. Proceedings of the $8^{\text {th }}$ International Workshop on Agent Theories, Architectures and Languages, ATAL-2001, Seattle, (2001)

13. Ferber, J., Gutknecht, O.: A meta-model for the analysis and design of organizations in multi-agent systems. In: Proceedings of the Third International Conference on MultiAgent Systems (ICMAS'98), IEEE Computer Society, (1998)

14. Frei, C., Faltings, B.: A Dynamic Hierarchy of Intelligent Agents for Network Management. In: Proceedings of 2nd International Workshop on Intelligent Agents for Telecommunications Applications (IATA'98), Paris, France (1998) 1-16

15. Jonker, C., Klusch, M., Treur, J.: Design of Collaborative Information Agents, Cooperative Information Agents IV. In: Klusch M., Kerschberg, L. (eds.): Cooperative Information Agents IV. LNAI 1860, Springer-Verlag (2000) $262-283$

16. Omicini, A.: SODA: Societies and Infrastructures in the Analysis and Design of Agentbased Systems. In: Ciancarini P., Wooldridge, M. (eds.): Agent-Oriented Software Engineering, LNCS 1957, Springer-Verlag (2001)

17. Ossowski, S.: Co-ordination in Artificial Agent Societies, LNAI 1535, Springer (1998)

18. Preece, A., Hui K., Gray, P.: KRAFT: Supporting Virtual Organisations through Knowledge Fusion. In: Finin T., Grosof B. (Eds): Artificial Intelligence for Electronic Commerce: Papers from the AAAI-99 Workshop, AAAI Press, (1999) 33-38.

19. Rocha, A.P., Oliveira, E.: An Electronic Market Architecture for the formation of Virtual Enterprises.Proceedings of PRO-VE'99 IFIP/PRODNET Conference on Infrastructures for Industrial Virtual Enterprises, Porto, October (1999)

20. Second Workshop on Norms and Institutions, Autonomous Agents, Montreal, http://ccs.mit.edu/dell/aa2001, (2001).

21. Tsvetovat, M., Sycara, K., Chen, Y., Ying, J.: Customer Coalition in Electronic Markets. In: Dignum, F., Cortés, U. (Eds.): Agent-Mediated Electronic Commerce III, LNAI 2003 (2001) 121-138

22. Wooldridge, M., Jennings, N., Kinny, D.: The Gaia Methodology for Agent-Orient Analysis and Design. Autonomous Agents and Multi-Agent Systems, 3(3) (2000) 285312

23. Zambonelli, F., Jennings, N., Omicini, A., Wooldridge, M.: Agent-Oriented Software Engineering for Internet Applications. In: A. Omicini, Zambonelli, F., Klusch, M., Tolkdorf, R. (eds.): Coordination of Internet Agents: Models, Technologies, and Applications. Springer-Verlag (2001) 326 - 346 